PAPER

\title{
Medial temporal lobe atrophy and white matter hyperintensities are associated with mild cognitive deficits in non-disabled elderly people: the LADIS study
}

\author{
W M van der Flier, E C W van Straaten, F Barkhof, J M Ferro, L Pantoni, A M Basile, D Inzitari, \\ T Erkinjuntti, L O Wahlund, E Rostrup, R Schmidt, F Fazekas, P Scheltens, on behalf of the LADIS \\ study group
}

J Neurol Neurosurg Psychiatry 2005;76:1497-1500. doi: 10.1136/jnnp.2005.064998

See end of article for authors' affiliations

.....................

Correspondence to: Dr Wiesje $M$ van der Flier, Department of Neurology and Alzheimer Centre, Vrije Universiteit Medical Centre, PO Box 7057 , 1007 MB Amsterdam, Netherlands; wm.vdflier@ vumc.nl

Received 7 February 2005 In revised form 24 February 2005

Accepted 24 March 2005

A list of participating centres is given in the appendix.
Objective: To assess the associations of medial temporal lobe atrophy (MTA) and white matter hyperintensities (WMH) with cognitive function in a large group of independently functioning elderly people.

Methods: Data were drawn from the multicentre, multinational leukoaraiosis and disability (LADIS) project which is studying prospectively the role of WMH as an independent predictor of the transition to disability in non-disabled elderly people. In all, 639 participants were enrolled in the LADIS study. For the present analysis, data on 581 subjects were available. Cognitive function was assessed by the mini-mental state examination (MMSE). Visual ratings of WMH and MTA were undertaken on magnetic resonance images (MRI).

Results: The presence of either severe WMH or MTA was associated with a modest but non-significant increase in frequency of mild cognitive deficits (severe $\mathrm{WMH}$ : odds ratio $(\mathrm{OR})=1.9(95 \%$ confidence interval (Cl), 1.0 to 3.7); MTA present: $\mathrm{OR}=1.5(95 \% \mathrm{Cl}, 0.8$ to 2.8$))$. However, subjects with the combination of MTA and severe WMH had a more than fourfold increase in frequency of mild cognitive deficits $(O R=4.1(95 \% \mathrm{Cl}, 2.3$ to 7.4$))$. Analysis of variance with post hoc Bonferroni $t$ tests showed that subjects with both MTA and severe WMH performed worse on MMSE than those with either no MRI abnormality or a single MRI abnormality $(p<0.05)$.

Conclusions: These results provide further evidence for the combined involvement of both Alzheimer type pathology and vascular pathology in the earliest stages of cognitive decline and suggest an additive effect of WMH and MTA.
$\mathrm{T}$ here is a growing body of evidence that in addition to the classical "Alzheimer type" neuropathology of senile plaques and neurofibrillary tangles, vascular pathology also plays a role in the neurodegenerative processes leading to cognitive impairment in old age. ${ }^{12}$ On magnetic resonance imaging (MRI), medial temporal lobe atrophy (MTA) presumably reflects Alzheimer type pathology, whereas white matter hyperintensities (WMH) are probably of vascular origin. $^{34}$

MTA and WMH often coexist in dementia. ${ }^{5}$ However, the role of the combination of these MRI abnormalities in the early stages of cognitive decline is not known. If MTA and WMH could be shown to influence cognitive function jointly in non-disabled elderly people, this would provide further evidence for the early involvement of both Alzheimer type pathology and vascular pathology in determining cognitive impairment. In this study, we therefore assessed the associations of MTA and WMH with cognitive function in a large group of independently functioning elderly people.

\section{METHODS}

Data were drawn from the multicentre and multinational leukoaraiosis and disability (LADIS) project which is studying prospectively the role of WMH as an independent predictor of the transition to disability in non-disabled elderly people. The design of the LADIS project has been fully described elsewhere. ${ }^{7}$ In short, 639 elderly subjects who had no or only mild disability in their instrumental activities of daily living (IADL) and who were stratified for WMH severity were enrolled between 18 July 2001 and 31 January 2003. Subjects presented with complaints including mild cognitive or motor disturbances, minor cerebral events, mood alterations, other neurological problems, or in whom WMH were incidentally identified on brain imaging. Subjects participating as controls in other studies and volunteers were also included. To be included, subjects had to meet the following criteria:

- age between 65 and 84 years;

- WMH on MRI of any degree, according to the categorisation into the three severity classes of a revised version of the Fazekas scale ${ }^{8}$;

- no or only mild disability as determined by the IADL scale";

- presence of a regularly contactable informant;

- agreement to sign informed consent.

Exclusion criteria were: the likelihood of dropping out because of the presence of severe illnesses (cardiac, hepatic, or renal failure, cancer, or other relevant systemic diseases); severe unrelated neurological diseases; leucoencephalopathy of non-vascular origin (immunological-demyelinating, metabolic, toxic, infectious, other); severe psychiatric disorders;

Abbreviations: IADL, instrumental activities of daily living; LADIS, leukoaraiosis and disability project; MMSE, mini-mental state examination; MTA, medial temporal lobe atrophy; WMH, white matter hyperintensities 
Table 1 Odds ratios for mild cognitive deficits dependent on medial temporal lobe atrophy and white matter hyperintensities

\begin{tabular}{lllll}
\hline Medial temporal lobe atrophy & Absent & Absent & Present & Present \\
White matter hyperintensities & Mild & Severe & Mild & Severe \\
Good cognitive function $(\mathrm{n})$ & 302 & 56 & 82 & 46 \\
Mild cognitive deficits $(\mathrm{n})$ & 40 & 14 & 16 & 25 \\
Crude odds ratio $(95 \% \mathrm{Cl})$ & 1.0 (reference) & $1.9(1.0$ to 3.7$)$ & $1.5(0.8$ to 2.8$)$ & 4.1 (2.3 to 7.4$)$ \\
Adjusted odds ratio $(95 \% \mathrm{Cl})$ & 1.0 (reference) & $1.8(0.9$ to 3.9$)$ & $1.4(0.7$ to 2.8$)$ & 4.6 (2.3 to 9.0$)$
\end{tabular}

Data are presented as odds ratios and their $95 \%$ confidence intervals (CI). Adjusted odds ratios are corrected for age, sex, education, and presence of stroke and/or lacunes.

MMSE, mini-mental state examination; MTA, medial temporal lobe atrophy; WMH, white matter hyperintensities.

inability to give informed consent; and inability or refusal to undergo cerebral MRI. Subjects are currently followed up for three years with repeated clinical and MRI studies. The present paper is based on the baseline data of the LADIS project.

All subjects were assessed using an extensive set of clinical and functional tests including global functioning, and cognitive, motor, psychiatric, and quality of life measures. For the present study, general cognitive function was assessed by the mini-mental state examination (MMSE). ${ }^{10}$ Subjects with an MMSE score of 26 or more were considered to have "good cognitive function," whereas those with an MMSE score below 26 were considered to have "mild cognitive deficit."

All subjects were studied by MRI following a standard protocol, and scans were collected centrally at the Image Analysis Centre (IAC) of the Vrije Universiteit Medical Centre, Amsterdam. The MR protocol included Tl weighted three dimensional MPRAGE (magnetisation prepared rapid acquisition gradient echo), axial T2, and FLAIR (fluid attenuated inversion recovery) sequences. Visual ratings of WMH and MTA were carried out centrally by a single rater (ECWvS) who was blind to clinical details. The degree of WMH severity was rated visually on axial FLAIR images using the Fazekas scale as grade 1 (punctate), grade 2 (early confluent), or grade 3 (confluent). ${ }^{8}$ Visual rating of MTA was done on $\mathrm{Tl}$ weighted images resliced in the coronal plane according to the $0-4$ point Scheltens scale. ${ }^{11}$ For 32 subjects, no Tl weighted scan was available and in 25 subjects, the quality of the Tl weighted scan did not allow MTA scoring. Thus 581 subjects participated in the present study. To determine the intrarater reliability, 18 scans were scored twice for WMH and 15 scans for MTA (weighted Cohen's $\kappa$ for $\mathrm{WMH}=0.84$ and for MTA $=0.85$ ).

For statistical analysis, both MRI measures were dichotomised (WMH grade 1 and $2=$ mild $W M H$, grade $3=$ severe WMH; MTA score $0 / 1=$ no atrophy, MTA score $2-4=$ atrophy). Using both dichotomised MRI measures, we computed a new four level categorical "MTA $\times W M H$ " variable. Crude odds ratios (OR) were calculated by cross tabulation. In addition, we used logistic regression analysis to correct for age, sex, education, and the presence of lacunes or stroke. Analysis of variance (ANOVA) with post hoc Bonferroni $t$ tests was used to compare differences in MMSE score according to the presence of MTA or severe WMH or both.

\section{RESULTS}

There were 581 subjects ( $54 \%$ female). Their mean (SD) age was 74 (5) years; their years of education, 10 (4); and their MMSE score, 27 (2). The majority of subjects had good cognitive function $(n=486$, defined as MMSE $\geqslant 26)$, but there were 95 subjects with mild cognitive deficits (MMSE $<26$ ). There was a predominance of women among the subjects with an MMSE score below $26\left(\chi^{2}=3.8, p=0.05\right)$, and they had received less education $(t=7.1, \mathrm{p}<0.001)$.

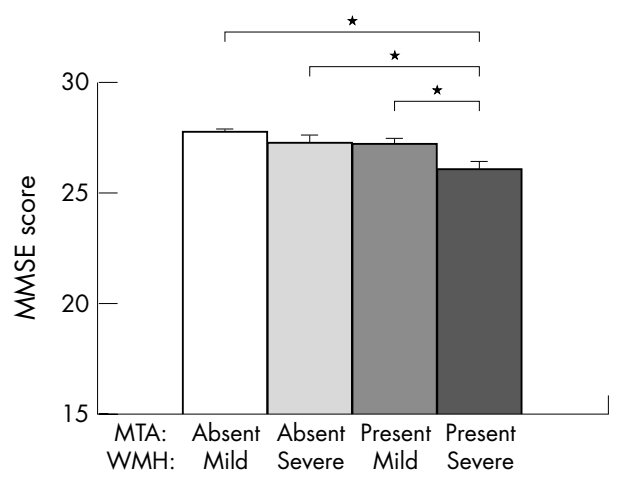

Figure 1 Mean mini-mental state examination (MMSE) score according to the presence of medial temporal lobe atrophy (MTA) and age related white matter hyperintensities (WMH). The group with both MTA and severe WMH had a lower MMSE score than the groups with a single MRI abnormality or no abnormality $(p<0.05)$.

There was a significant correlation between the presence of severe WMH and MTA (Spearman's $r=0.27, \mathrm{p}<0.001$ ). The presence of either severe WMH or MTA was associated with a modest but non-significant increase in frequency of mild cognitive deficits (severe WMH, $\mathrm{p}=0.06$; MTA present, $\mathrm{p}=0.23$, see table 1 ). However, subjects with the combination of MTA and severe WMH had a more than fourfold increase in the frequency of mild cognitive deficits $(p<0.001)$. These risk estimates did not change essentially when age, sex, education, and the presence of stroke or lacunes were entered as covariates. Formal testing in a logistic regression model incorporating the interaction term between MTA and WMH showed that there was no statistical interaction between MTA and WMH.

In an additional analysis, we compared the MMSE score of the four "MTA $\times \mathrm{WMH}^{\prime}$ " groups using ANOVA. There was a difference in MMSE score between groups $(p<0.001$, fig 1$)$. Post hoc analyses using Bonferroni $t$ tests showed that the group with both MTA and severe WMH performed significantly worse than the other three groups of subjects with either no MRI abnormality or a single abnormality $(\mathrm{p}<0.05)$. The presence of either MTA but no severe WMH, or severe WMH but no MTA was not associated with a significantly lower MMSE score.

\section{DISCUSSION}

We showed that a combination of MTA and severe WMH was associated with a more than fourfold increase in the frequency of mild cognitive deficits in a large group of nondisabled elderly people. By contrast, mild cognitive deficits were not more frequent in the groups of patients with a single MRI abnormality (either MTA or severe WMH). These results suggest the combined involvement of both Alzheimer type pathology and vascular pathology in the earliest stages of cognitive decline. 
Former studies (both necropsy and MRI) reported that Alzheimer type pathology and vascular pathology often coexist in patients with dementia. ${ }^{1256}$ The present study extends these findings, as we observed in vivo using MRI that even in non-disabled elderly people the combination of MTA and WMH was already associated with decreased cognitive performance. These findings provide further evidence for the threshold theory, which stipulates that Alzheimer type pathology and cerebrovascular pathology summate until a threshold is reached and clinical dementia ensues. ${ }^{12}$

In seeming contrast to earlier studies, ${ }^{513-15}$ the presence of MTA alone was not significantly associated with cognitive impairment. The specific characteristics of the LADIS study sample probably account for this finding. Subjects were included on the basis that they had WMH on MRI in combination with absence of disability, resulting in a study population that is biased towards excessive vascular pathology and relatively little Alzheimer type pathology. However, even in this population, where cognitive impairment would be expected to be of vascular origin, the association between WMH and cognitive impairment could only be fully appreciated if MTA was also taken into account.

A possible limitation of this study includes the use of dichotomised rather than continuous measures of MTA and WMH. In addition, we used the MMSE, which is a rather crude measure of cognitive function. It is conceivable that the use of continuous MRI variables and a more sensitive cognitive test would have yielded more striking results. However, we feel that our simple approach enhances the clinical value of our results, as the MMSE is one of the most widely used cognitive tests. In addition, contrasting with sophisticated, but time intensive neuroimaging measures, simple ratings of MTA and WMH can be used in clinical practice.

These results may have important clinical implications. Memory clinics often evaluate patients who present with subjective memory complaints and severe WMH on MRI, but who have no detectable cognitive deficits. The present study suggests that if such patients have additional MTA they may be at increased risk of subtle cognitive impairment. Should further studies using a longitudinal design corroborate our finding that the combination of WMH and MTA is indeed predictive of subsequent cognitive decline, the combination of these simple MRI markers may be a useful tool in identifying subjects at risk of dementia. Whether this combination of markers predicts dementia at follow up in the present sample will be the subject of further study.

\section{ACKNOWLEDGEMENTS}

The LADIS study is supported by the European Union within the V European Framework Programme "Quality of life and management of living resources" (1998-2002), contract No QLRT-2000-00446 as a concerted action.

\footnotetext{
Authors' affiliations

W M van der Flier, E C W van Straaten, P Scheltens, Department of Neurology and Alzheimer Centre, Vrije Universiteit Medical Centre, Amsterdam, Netherlands

F Barkhof, Department of Radiology and Image Analysis Centre (IAC), Vrije Universiteit Medical Centre

J M Ferro, Serviço de Neurologia, Centro de Estudos Egas Moniz, Hospital de Santa Maria, Lisbon, Portugal

L Pantoni, A M Basile, D Inzitari, Department of Neurological and Psychiatric Sciences, University of Florence, Florence, Italy T Erkinjuntti, Memory Research Unit, Department of Clinical Neurosciences, Helsinki University, Helsinki, Finland L O Wahlund, Neurotec department, Section of Clinical Geriatrics, Karolinska Universitetssjukhuset, Huddinge University Hospital, Huddinge, Sweden

E Rostrup, Memory Disorders Research Unit, Department of Neurology, Copenhagen University Hospital, Copenhagen, Denmark
}

R Schmidt, F Fazekas, Department of Neurology and MRI Institute, Medical University, Graz, Austria

Competing interests: Philip Scheltens is an associate editor of JNNP but had no role in reviewing or accepting this paper.

\section{APPENDIX}

LIST OF PARTICIPATING CENTRES AND PERSONNEL Helsinki, Finland (Memory Research Unit, Department of Clinical Neurosciences, Helsinki University): Timo Erkinjuntti MD, PhD, Tarja Pohjasvaara MD, PhD, Pia Pihanen MD, Raija Ylikoski PhD, Hanna Jokinen LPsych, Meija-Marjut Somerkoski MPsych, Riitta Mäntylä MD, PhD, Oili Salonen MD, PhD; Graz, Austria (Department of Neurology and MRI Institute, Medical University): Franz Fazekas MD, Reinhold Schmidt MD, Stefan Ropele PhD, Alexandra Seewann MD, Katja Petrovic MagPsychol, Ulrike Garmehi; Lisbon, Portugal (Serviço de Neurologia, Centro de Estudos Egas Moniz, Hospital de Santa Maria): José M Ferro MD, PhD, Ana Verdelho MD, Sofia Madureira PsyD; Amsterdam, Netherlands (Department of Neurology, VU Medical Centre): Philip Scheltens MD, PhD, Ilse van Straaten MD, Alida Gouw MD, Wiesje van der Flier PhD, Frederik Barkhof MD, PhD; Goteborg, Sweden (Institute of Clinical Neuroscience, Goteborg University): Anders Wallin MD, PhD, Michael Jonsson MD, Karin Lind MD, Arto Nordlund PsyD, Sindre Rolstad PsyD, Kerstin Gustavsson RN; Huddinge, Sweden (Neurotec department, Section of Clinical Geriatrics, Karolinska Universitetssjukhuset): Lars-Olof Wahlund MD, $\mathrm{PhD}$, Militta Crisby MD, PhD, Anna Pettersson, physiotherapist, Kaarina Amberla PsyD; Paris, France (Department of Neurology, Hopital Lariboisiere): Hugues Chabriat MD, PhD, Ludovic Benoit MD, Karen Hernandez, Solene Pointeau, Annie Kurtz, Daniel Reizine MD; Mannheim, Germany (Department of Neurology, University of Heidelberg, Klinikum Mannheim): Michael Hennerici MD, Christian Blahak MD, Hansjorg Baezner MD, Martin Wiarda PsyD, Susanne Seip RN; Copenhagen, Denmark (Copenhagen University Hospital: Memory Disorders Research Unit, Department of Neurology, Rigshopitalet, and the Danish Magnetic Resonance Research Centre, Hvidovre Hospital): Gunhild Waldemar MD, DMSc, Egill Rostrup MD, MSc, Charlotte Ryberg, Tim Dyrby; Newcastleupon-Tyne, UK (Institute for Ageing and Health, University of Newcastle): John O'Brien DM, Sanjeet Pakrasi MRCPsych, Thais Minnet PhD, Michael Firbank PhD, Jenny Dean PhD, Pascale Harrison BSc, Philip English DCR.

The Coordinating centre is in Florence, Italy (Department of Neurological and Psychiatric Sciences, University of Florence): Domenico Inzitari MD (Study Coordinator); Leonardo Pantoni MD, PhD, Anna Maria Basile MD, Michela Simoni MD, Giovanni Pracucci MD, Monica Martini MD, Luciano Bartolini PhD, Emilia Salvadori PhD, Marco Moretti MD, Mario Mascalchi MD, PhD.

The LADIS Steering Committee is formed by Domenico Inzitari MD (study coordinator), Timo Erkinjuntti MD, PhD, Philip Scheltens MD, PhD, Marieke Visser MD, PhD, and Kjell Asplund MD, PhD.

\section{REFERENCES}

1 Snowdon DA, Greiner LH, Mortimer JA, et al. Brain infarction and the clinical expression of Alzheimer disease. The Nun Study. JAMA 1997:277:813-17.

2 Pathological correlates of late-onset dementia in a multicentre, communitybased population in England and Wales. Neuropathology Group of the Medical Research Council Cognitive Function and Ageing Study (MRC CFAS). Lancet 2001:357:169-75.

3 Gosche KM, Mortimer JA, Smith CD, et al. Hippocampal volume as an index of Alzheimer neuropathology: Findings from the Nun Study. Neurology 2002;58:1476-82.

4 Pantoni L, Garcia JH. Pathogenesis of leukoaraiosis: a review. Stroke 1997:28:652-9.

5 van der Flier WM, Middelkoop HA, Weverling-Rijnsburger AW, et al. Interaction of medial temporal lobe atrophy and white matter hyperintensities in AD. Neurology 2004;62:1862-4. 
6 de Leeuw FE, Barkhof $F$, Scheltens $P$. White matter lesions and hippocampal atrophy in Alzheimer's disease. Neurology 2004;62:310-12.

7 Pantoni L, Basile AM, Pracucci G, et al. Impact of age-related cerebral white matter changes on the transition to disability - the LADIS study: rationale, design and methodology. Neuroepidemiology $2005 \cdot 24 \cdot 51-62$

8 Fazekas F, Chawluk JB, Alavi A, et al. MR signal abnormalities at $1.5 \mathrm{~T}$ in Alzheimer's dementia and normal aging. Am J Roentgenol 1987;149:351-6.

9 Lawton MP, Brody EM. Assessment of older people: self-maintaining and instrumental activities of daily living. Gerontologist 1969:9:179-86.

10 Folstein MF, Folstein SE, McHugh PR. Mini-mental state. A practical method for grading the cognitive state of patients for the clinician. $J$ Psychiatr Res 1975; 12:189-98.
11 Scheltens $\mathbf{P}$, Launer $\sqcup$, Barkhof $F$, et al. Visual assessment of medial temporal lobe atrophy on magnetic resonance imaging: interobserver reliability. J Neurol 1995;242:557-60.

12 Esiri MM, Nagy Z, Smith MZ, et al. Cerebrovascular disease and threshold for dementia in the early stages of Alzheimer's disease. Lancet 1999-354:919-20.

13 Chetelat G, Baron JC. Early diagnosis of Alzheimer's disease: contribution of structural neuroimaging. Neuroimage 2003;18:525-41.

14 Petersen RC, Jack CRJ, Xu YC, et al. Memory and MRI-based hippocampal volumes in aging and AD. Neurology 2000:54:581-7.

15 Mungas D, Jagust WJ, Reed BR, et al. MRI predictors of cognition in subcortical ischemic vascular disease and Alzheimer's disease. Neurology 2001;57:2229-35

\section{NEUROLOGICAL PICTURE}

Hemifacial spasm, neuralgia, and syncope due to cranial nerve compression in a patient with vertebral artery ectasia

A 42 year old woman with a history of an incompletely remitted peripheral left facial nerve palsy that was followed by clusters of hemifacial spasm and paroxysms of lancinating pain located behind the left ear and radiating to the ipsilateral eye, the neck, or shoulder, was admitted for reevaluation due to additionally manifested short lasting syncope-like episodes of increasing frequency. Beneath a mild residual facial nerve palsy no further focal findings were documented. Extensive cardiologic workup revealed only a positive tilt table test, indicating an intense vagovasal reaction.

Re-evaluation of previously performed imaging examinations focused on an already angiographically described tortuous course of the vetrebrobasilar arteries (panel A), which compressed the left lateral surface of the medulla, pons, and pontomedullar junction, as also demonstrated on MRI (panel B). Based on the documented vagovasal reaction we assumed an affection of the lower cranial nerves and additionally performed ultra thin MRI. In this way we could impressively demonstrate a severe compression of the proximal stem of the cranial nerves IX and $X$ (panel D), and the root entry zone of the facial nerve on the left (panel C). Owing to the escalation of symptoms the patient underwent microvascular decompressive surgery with interposition of a muscle graft. Two years later she remains asymptomatic. A repeated tilt table test was this time negative.

This is an uncommon case of neurovascular compression, ${ }^{1}$ characterised by known, however previously not in this combination, described features such as hemifacial spasms, variant glossopharyngeal neuralgia, and syncope, ${ }^{2-5}$ which all ceased after surgery.

K Spengos, G Tsivgoulis, G Stouraitis, D Vassilopoulos Department of Neurology, University of Athens, Athens, Greece
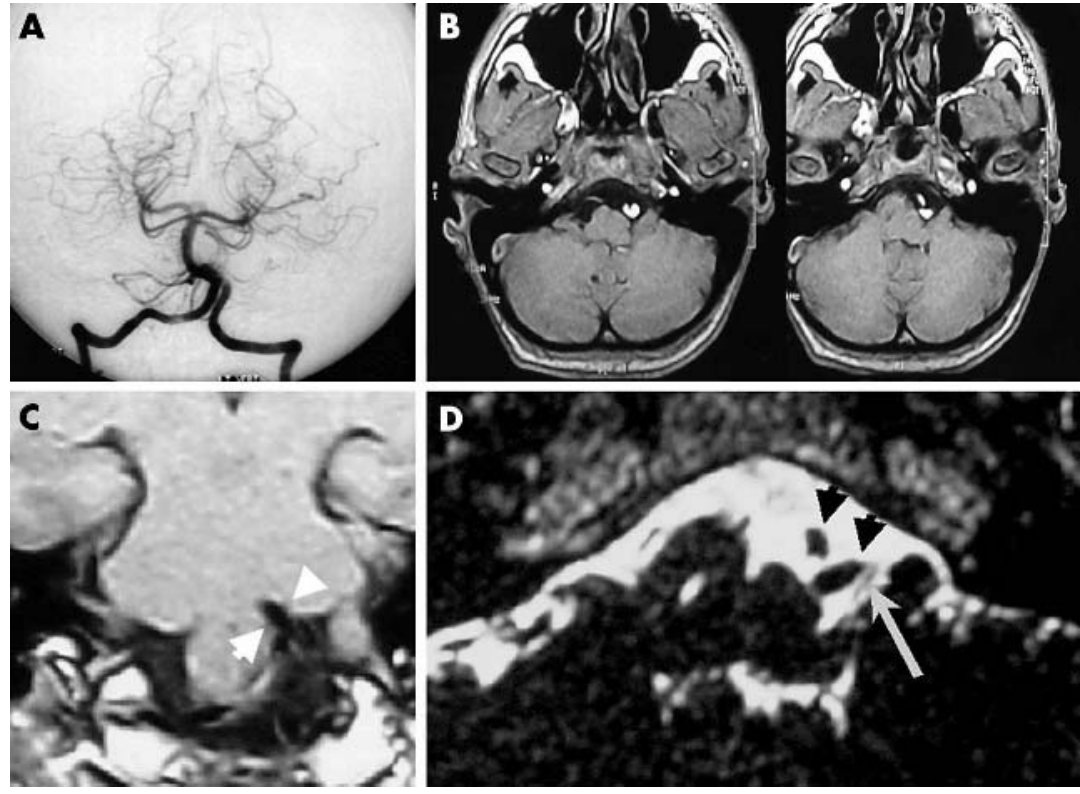

(A) Superimposed projection of the digital subtraction angiograms of both vertebral arteries demonstrates impressively the tortuous course of the vertebrobasilar vessels. (B) Axial T1-GRE weighted MRI sequences demonstrating the tortuous course of both vertebral arteries and their compressing effect on the left frontolateral surface of the pontomedullar junction, the pons, and the cerebellar peduncles. (C) Frontal T1 weighted MRI slice showing the compressing effect of both vertebral arteries (white arrow heads) on the root entry zone of the left facial nerve. (D) Ultra thin $(0.8 \mathrm{~mm}) \mathrm{M} 3 \mathrm{D} / \mathrm{FSE}$ weighted MRI slice showing the direct contact between the vertebral artery (black arrow head) and the IX/X cranial nerve (grey arrow).

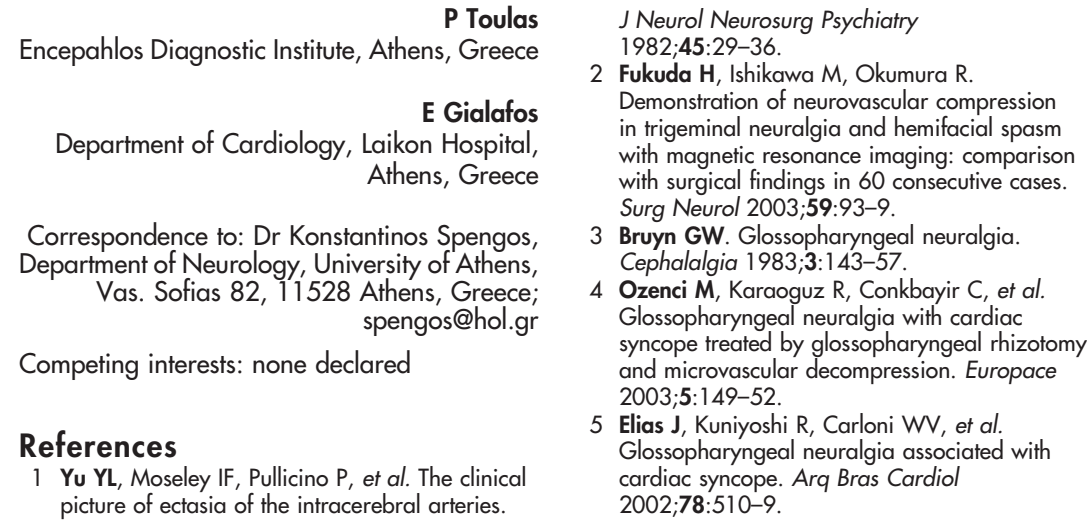
1982;45:29-36. spasm with magnetic resonance imaging: comparison Surg Neurol 2003.59:93-9.

Bruyn GW. Glossopharyngeal neuralgia. Cephalalgia 1983;3:143-57.

Glossopharynge syncope treated by glossopharyngeal rhizotomy 2003;5:149-52. 2002;78:510-9. 